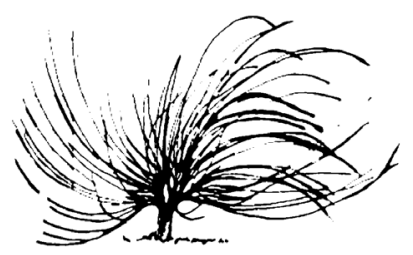

\title{
Adquisición de vocabulario productivo en contextos académicos superiores: análisis de su evolución a partir de un estudio longitudinal
}

\author{
Martín Gonzalo Zapico ${ }^{1}$ \\ Instituto de Formación Docente Continua San Luis \\ Argentina \\ athenspierre@gmail.com
}

\begin{abstract}
Resumen
El vocabulario, tanto en su dimensión productiva como receptiva, es un elemento fundamental a la hora de afrontar una carrera académica. En este marco, su medición y la posibilidad de entender cómo es su adquisición se tornan fundamentales, a efectos de delinear políticas concretas de intervención que ayuden a paliar el abandono, en particular el del primer año de muchas carreras de nivel superior. En este trabajo, se muestran los resultados de un análisis realizado a través del seguimiento durante cuatro años de un grupo de estudiantes a los cuales se les aplicó un test de vocabulario productivo cuatro veces, una a principio de cada año lectivo. Los resultados sugieren que la adquisición de vocabulario está fundamentada en la reacomodación de conceptos, semejante a una función exponencial.
\end{abstract}

\section{(c) (1) $\circledast \Theta$}

Recibido: 1 de febrero de 2018. Aprobado: 24 de setiembre de 2018.

http://dx.doi.org/10.15359/rep.13-2.6

1 Profesor de Letras recibido en la UNMDP. Actualmente, se desempeña como profesor responsable en el IFDC-San Luis y doctorando en Educación en la UNSL. Ha realizado numerosas publicaciones en revistas científicas, así como tres libros y asistido a numerosas reuniones científicas en los campos de educación, lingüística y psicología. 
Palabras claves: vocabulario, desempeño académico, abandono escolar, estudio longitudinal, educación superior.

\begin{abstract}
Vocabulary, both in its productive and receptive dimension, is a fundamental element when facing an academic major. In this context, its measurement and the possibility of understanding how it is acquired becomes fundamental in order to design concrete intervention policies that help to palliate dropouts, particularly the first year of many higher education majors. In this framework, the results of an analysis carried out through the four-year follow-up of a group of students who were applied a test of productive vocabulary four times, one at the beginning of each school year are shown. The results suggest that the acquisition of vocabulary is based on the rearrangement of concepts, similar to an exponential function.
\end{abstract}

Keywords: vocabulary, academic performance, school dropouts, longitudinal study, higher education

\title{
1. Estado del arte
}

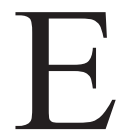

1 vocabulario como gran constructo puede ser analizado en dos planos diferentes: el de la recepción y el de la producción. El de la recepción se relaciona con las competencias que tiene un sujeto para interpretar un texto codificado mediante un sistema simbólico cualquiera (en lo que compete a educación, el idioma en el cual se comunican docente-alumno, así como el de los textos), a partir del reconocimiento de los relaciones sintagmáticas y paradigmáticas fundamentales que rige la gramática de una lengua. Por otro lado, el vocabulario productivo se refiere a la capacidad de un sujeto de producir enunciados que se adecuen a las normas de una lengua (en este ámbito entran la redacción, la capacidad de expresión verbal, la argumentación, entre otras). Si bien es cierto que en todo proceso comunicativo se requieren ambas, diversos test (Casso, 2010) ponen de manifiesto que el vocabulario productivo susbsume al receptivo, es decir, que es más fácil para un hablante o incluso un aprendiz leer un texto que producirlo. 
Para el caso de la competencia léxico-productiva y su relación con el ámbito académico, su importancia siempre ha sido señalada desde diversos ángulos. Desde una perspectiva general, al hablar de vocabulario (González y Martínez, 1998), se ha señalado la importancia que adquiere en los procesos de comunicación del alumno tanto con las autoridades como con sus pares. Este énfasis en la comunicación está fundamentado, principalmente, en que el éxito en la vida académica no se trata solo de instancias de evaluación formales, sino también del establecimiento de relaciones de comunicación efectivas con una enorme cantidad de actores sociales (Álvarez Castrillo y Diez-Itza, 2000; Ocares et al., 2013). Lo anterior qiere decir que un lexicón lo suficientemente amplio permitirá a su usuario realizar con éxito tareas del tipo solicitar o dar información, expresar acuerdo o desacuerdo e incluso organizar sus labores.

En relación con la capacidad de lectura de textos académicos (Suárez, Moreno y Godoy, 2010), se ha encontrado que la mayoría de inconvenientes en cuanto a su lectura e interpretación están vinculados al vocabulario, de dos formas: por un lado, los estudiantes aducen no conocer una gran cantidad de palabras que aparecen en dichos textos; por otro, no pueden dar sentido a una cuantiosa cantidad de oraciones que allí aparecen, lo cual imposibilita la construcción de ideas en torno al significado del texto. Estas dificultades de comprensión, naturalmente, derivan en muchos fracasos académicos en las instancias evaluativas (Nieto, 2005), lo cual suele conllevar a la frustración del alumno y la consecuente duda sobre las propias capacidades.

Sobre la capacidad de escritura académica (Carlino, 2001; Carlino, 2007) se ha hecho foco en las dificultades que conlleva la adaptación a este tipo de texto, al cual los alumnos se han visto pocas veces expuestos en su trayectoria de nivel secundario. En este marco, el vocabulario se vuelve necesario no tanto como contenido sino como forma. Las deficiencias en la capacidad de producción de textos con formato académico (ensayos, informes, trabajos prácticos monográficos) no se relacionan con una ausencia de palabras en sentido estricto, sino con una falta de palabras o frases que permitan dar la forma requerida a las ideas.

Específicamente la competencia léxico-productiva y su incidencia en el desempeño académico de forma directa, a través del análisis de resultados obtenidos en instancias de evaluación (Zapico, 2016, 2016b y 2017), ha revelado correlaciones estadísticamente significativas entre 
la capacidad léxica y el desempeño académico, poniendo de relieve la importancia del dominio del vocabulario para optimizar las habilidades de producción oral y escrita en contextos de educación superior.

Todos estos indicios colocan en primer plano la importancia de generar y promover espacios de consolidación del vocabulario, a fin de paliar las problemáticas de deserción que se observan en la matrícula tanto en el nivel nacional y regional como mundial. La importancia de trabajar sobre esta variable (Zapico, 2017b) reside en que, de las cuatro más relevantes que inciden en el desempeño (motivación y autoestima, nivel socioeconómico, desempeño previo y vocabulario), es la única en que se puede incidir de manera directa y en un lapso breve.

En cuanto a la forma en que se adquiere el vocabulario, hay una gran cantidad de estudios que abordan la temática, pero, en su mayoría, dichos trabajos se basan en el aprendizaje siempre de una segunda lengua (Sigüan, 2001; Moya Guijarro, 2003; Oster, 2009) o, en su defecto, tratan de describir como se da el proceso de adquisición del lenguaje en etapas del desarrollo madurativo de los niños (Manso y Ballesteros, 2003). A esta generalidad se encuentran excepciones como el estudio de Martínez Fuentes (1996), que busca observar el papel del temperamento en la adquisición del vocabulario, pero sin resultados concluyentes. Concretamente para la adquisición de vocabulario de manera activa, y en el nivel de educación superior-universitario, no hay estudios disponibles.

Con la idea de tratar de comprender el fenómeno de adquisición del vocabulario a lo largo de una carrera, se realizó un estudio de análisis longitudinal, de naturaleza correlacional-descriptiva. Por esta última, no se planteó ninguna hipótesis en concreto.

\section{Metodología}

\subsection{Instrumento empleado}

Se utilizó la prueba de vocabulario productivo Z-Test de sinónimos, para cuya elaboración se tomaron las Normas de Producción de Atributos Semánticos en Castellano Rioplatense (Vivas, Comesaña, García Coni, Vivas y Yerro, 2013), a partir de las cuales se obtuvieron 100 palabras de alta frecuencia que poseen sinónimos, según WordReference diccionario de sinónimos (2016). Luego, a través de la comparación con la base CREA de las 1000 palabras más frecuentes de la Real Academia Española (2016) y el juicio de pares especialistas en 
el área empleando el método de agregados individuales (5 expertos), se llegó a una lista final de 40 palabras, de la que solo se tuvieron en cuenta los $100 \%$ de coincidencia favorable, para una mayor solidez del instrumento. Una vez obtenidos estos 40 ítems, se realizó una prueba de IVC con un valor de $.99, \mathrm{~N}=5 \mathrm{y} \mathrm{N}_{\mathrm{e}}=5$, al ser expertos diferentes a los empleados en la etapa anterior del proceso y obtener una puntuación de 1 .

Para la validación externa, se realizó una prueba piloto $\mathrm{N}=32$, en la que se contrastaron los resultados obtenidos en el Z-Test con los derivados de evaluaciones formales del sistema de educación. Dado que hay un amplio acuerdo entre la relación del vocabulario de amplio espectro con el desempeño académico, puesto que en este se ven involucradas tareas de lectura y escritura, se esperaba observar correlaciones entre ambos resultados. El resultado de la prueba ANOVA, con un p.crítico $=0.05$ y F $=2.63$, fue de $\mathrm{p}=0.04$, mostrando una relación sólida entre la competencia léxica y el desempeño académico.

Para el cálculo del coeficiente de validez interna se contrastaron las puntuaciones obtenidas en la prueba piloto $\mathrm{N}=124$ con las logradas por los mismos sujetos en el Test de denominación de Boston (Serrano et al. 2001). Aplicando una estadística de correlación lineal de Pearson, se obtuvo $r=0.78$, que es significativamente alto, si se considera que el test mencionado mide el vocabulario en sus dos dimensiones. Si a esto le sumamos la controversia histórica y actual sobre la necesidad o no de dividir dichas dimensiones, nos encontramos ante una validez interna que es al menos suficiente.

Dada la naturaleza de lo que se mide, el vocabulario, se descartó la posibilidad de utilizar un método de test-retest, puesto que había alto riesgo de que los sujetos desarrollaran aprendizaje. Por ello, se aplicó la técnica conocida como formas paralelas, en la que se emplearon 2 versiones equivalentes del Z-Test, con una distancia de una hora entre ambas; se encontró un $r$ pearson $=0.86$, lo cual indica que prácticamente el $90 \%$ de la varianza del test no se debe al error.

En este test, las puntuaciones obtenibles pueden ir desde 0 a 40 y se delimitan 4 grupos de forma orientativa: A ( $>30$ alta competencia léxica), B $(20<30$ buena competencia léxica $), C(10<20$ regular competencia léxica) y $\mathrm{D}(<10$ baja competencia). 


\subsection{Muestra}

Se obtuvo un $\mathrm{N}=53$ ( $44 \%$ hombres y $64 \%$ mujeres, de entre 18 y 25 años, con $\left.\mathrm{x}=19,8 ; \mathrm{s}=2,43 ; \mathrm{s}^{2}=5,81\right)$, alumnos que transitaron 4 años provenientes de diversas carreras universitarias y de nivel superior, cuyos planes de estudio estaban estipulados en 4-5 años. A esta muestra se llegó a partir de un $\mathrm{N}$ inicial de 165 sujetos posibles, de los cuales solo a 53 se les pudo dar seguimiento, dado que únicamente ese número llegó al cuarto año de su respectiva carrera.

\subsection{Procedimiento}

Una vez obtenido el consentimiento oral que fue posteriormente respaldado por su versión escrita, se procedió a la administración del Z-Test. Este se llevó a cabo de manera individual en el espacio de trabajo del docente-investigador, bajo condiciones óptimas de iluminación y sonido, sin distractor alguno. El tiempo de administración fue de 30 minutos, aunque ningún sujeto superó dicha medida. Este mismo procedimiento fue realizado un total de 4 veces, a principios de los ciclos lectivos de los años 2014, 2015, 2016 y 2017.

\subsection{Análisis}

Una vez obtenidas las puntuaciones de todos los test durante los 4 años, se realizó una comparación de medias interanual junto con sus correspondientes medidas de dispersión. Se hizo, además, una medida del coeficiente de variación de los sujetos de forma tanto individual como grupal, a lo largo de los 4 años, y se estableció la función matemática de mejor ajuste para dicho coeficiente.

\section{Resultados y discusión}

A continuación, se muestran los resultados del análisis, empezando por un panorama general de la distribución de las puntuaciones a lo largo de los 4 años, junto con las líneas que la representan. 


\section{Figura 1.}

\section{Distribución de puntuaciones del test de vocabulario}

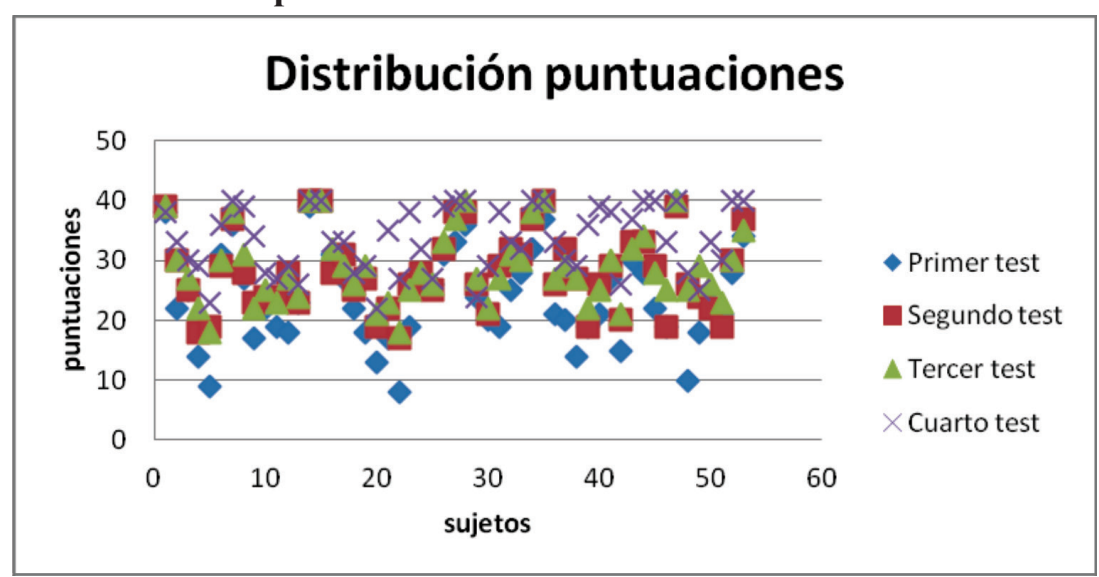

Fuente: Datos del autor.

A simple vista, podemos observar la evolución de las puntuaciones a lo largo de las distintas instancias de aplicación del instrumento. $\mathrm{Al}$ contrastar las primeras puntuaciones con las últimas, encontramos una superposición de puntos mínima, que manifesta la ostensible mejora de puntuaciones. Por otro lado, en la segunda y tercera aplicación, la superposición de puntuaciones es considerable, fenómeno que analizaremos más adelante en el trabajo, a partir de la comparación de medias.

La clarificación de esta distribución puede verse de forma más nítida con las líneas de dispersión. 
Figura 2.

Distribución de puntuaciones del test de vocabulario presentadas en forma de onda

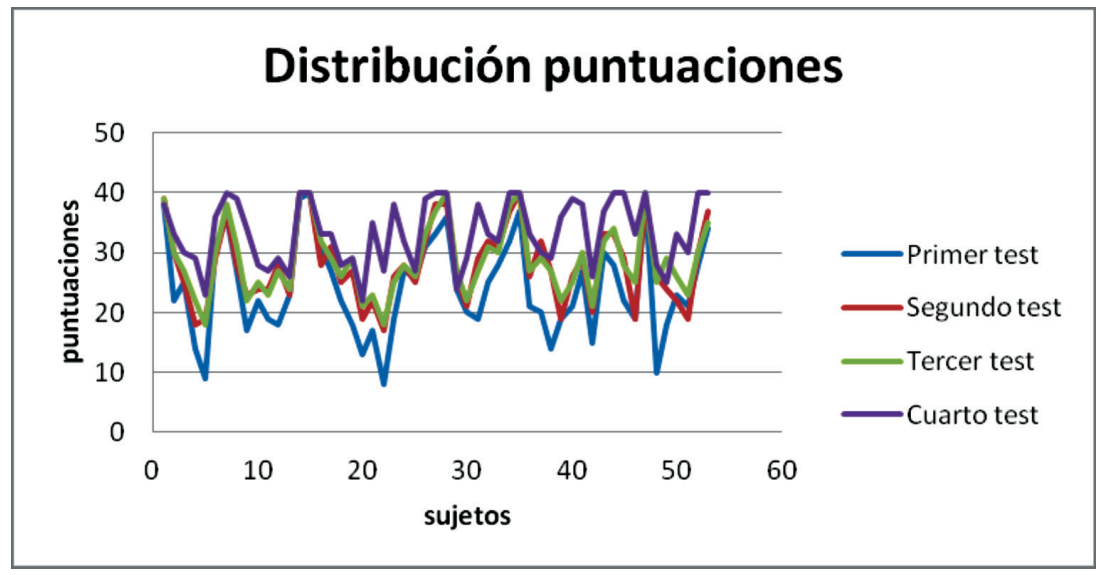

Fuente: Datos del autor.

Como marcamos anteriormente, en el segundo y tercer test, nos encontramos ante la superposición de puntuaciones, junto con una oposición marcada entre la primera y cuarta puntuación. Para empezar el trabajo pormenorizado, observemos la comparación interanual de medias, en la que se puede apreciar la evolución de la puntuación general de las pruebas obtenidas.

Tabla 1.

Comparación de medias entre años

\begin{tabular}{|c|c|c|c|c|}
\hline \multicolumn{5}{|c|}{ Comparación de medias interanual } \\
\hline Medidas/año & $\mathbf{A 1}$ & $\mathbf{A 2}$ & $\mathbf{A 3}$ & $\mathbf{A 4}$ \\
\hline $\mathrm{X}$ & 24,21 & 28,09 & 28,79 & 33,36 \\
\hline $\mathrm{X}$ & 19,00 & 19,00 & 27,00 & 40,00 \\
\hline $\mathrm{Me}$ & 23,00 & 28,00 & 28,00 & 33,00 \\
\hline $\mathrm{s} 2$ & 65,24 & 42,36 & 35,94 & 30,47 \\
\hline $\mathrm{s}$ & 8,08 & 6,51 & 5,99 & 5,52 \\
\hline
\end{tabular}

Fuente: Datos del autor. 
Lo primero que se pone en evidencia es que, efectivamente, a medida que pasaron los años, los sujetos fueron consolidando más su vocabulario. Esto, que de primera mano puede sonar a obviedad, da pie a preguntarse por qué sucede y de qué forma lo hace. Una primera idea, en consonancia con los estudios existentes sobre adquisición de vocabulario (Valdehíta, 2012; Valdehíta, 2013), estaría en afirmar que es la mera exposición constante a textos académicos que incrementa de forma lineal el repertorio léxico disponible. En primera instancia, parece ser bastante cierto, dado que no hay otra forma de acceder al conocimiento de vocablos, si no es por la exposición a ellos, sea de forma oral o escrita. Al hablar de exposición en estos casos, nos referimos a un trabajo con los textos, en el cual interviene una lectura más bien comprensiva. El problema contra esta opción es que, si bien la media de la puntuación obtenida por el conjunto aumentó año tras año, en el período $\mathrm{A} 2-\mathrm{A} 3$, prácticamente no hubo un incremento, expresando un estancamiento bastante marcado que, sin embargo, se dispara en el año 4 con un crecimiento igual de grande que de A1 a A2.

La hipótesis que surge en esta situación, bastante intuitiva, es que el primer año de carrera el estudiante se enfrenta a una enorme cantidad de información nueva, con formato, estilo de redacción y complejidad conceptual mucho más grande que aquella con la que trataba en el secundario. En caso de completar el primer año, y teniendo en cuenta que este suele ser el punto de mayor deserción académica en todas las carreras (Rodríguez et al., 2016), es lógico pensar que habrá consolidado todo un lexicón nuevo, suceso que se expresa en el sensible incremento de A1-A2.

Por otro lado, el crecimiento A3-A4 estaría guiado por lo que en psicología se ha llamado redes conceptuales (Galagovsky Kurman, 1993; Galagovsky Kurman, 1996), tema que siempre estuvo relacionado con los procesos de aprendizaje, en especial, desde la popularización del concepto de insight (Galagovsky Kurman, 2004), que describe el proceso a través del cual una gran cantidad de información semántica, desordenada o inconexa, muta de manera tal que pasa a estar ordenada y a adquirir sentido. Esta forma de aprendizaje, siempre presente como posibilidad de aprendizaje y de cuya existencia todo el tiempo ha habido acuerdo, vio finalmente confirmado su ser empírico primero por la neuropsicología (Palma y Cosmelli, 2008) y hace no mucho por la neurología (Falco y Kuz, 2016). De este modo, la consolidación de vocabulario en este período no estaría dada solamente por la exposición 
cada vez mayor a textos (dado que esta exposición es siempre constante, no debería darse el caso del estancamiento en los 2 años del medio), sino mayormente por la posibilidad de reordenar y reacomodar todos los conceptos estudiados en el trayecto de años de estudio. Esta idea se ve reforzada por la moda y la mediana, que también manifiestan un incremento muy marcado en el período A3-A4, cuando las puntuaciones del test empiezan a tender hacia los valores más altos. Otra prueba bastante sólida de cómo las puntuaciones empiezan a subir de forma marcada es la progresiva disminución de las medidas de dispersión, lo que indica que año tras año las puntuaciones recorren rangos menores y se acomodan cada vez más en las franjas altas del test.

Volviendo al período A2-A3, afirmamos que es un lapso de estancamiento, lo cual se corrobora al observar que no hay prácticamente cambio alguno en las puntuaciones medias y la mediana se mantiene exactamente en el mismo sitio. Dicho eso, hay un valor que llama la atención, por ser el único que fluctúa de forma considerable en este período, y es la moda. Esto parece indicar que hubo una porción de la muestra que el salto cuantitativo dado en A1-A2 logró en el período A2A3, lo cual se confirma al observar que tanto en A1 como en A2 la moda adquiere el valor de 19, dado que ni moda ni mediana variaron. Esto implica, además, que ese grupo, digamos rezagado, alcanzó puntuaciones más altas más tarde; el grupo que ya había llegado a esas puntuaciones o las mantuvo o, en muchos casos, obtuvo incluso una puntuación levemente más baja en A3 que en A2. Puede llegar a pensarse, entonces, que el insight es una especie de límite o frontera que es necesario cruzar para llegar a un aprendizaje significativo, tras el que haya una verdadera comprensión conceptual de lo que se estudia.

Ahora vamos a observar gráficamente la evolución de las puntuaciones, a la luz de establecer funciones de ajuste, empezando por la tendencia ya descripta anteriormente. 
Figura 3.

Línea de tendencia a través de los años

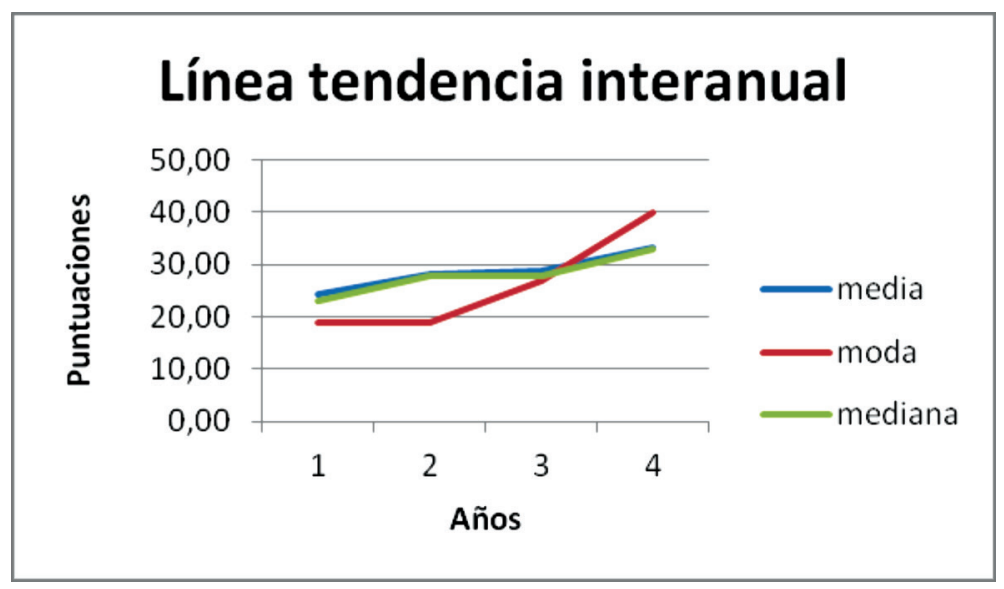

Fuente: Datos del autor.

Cada una de las líneas tomada por separado estaría definiendo distintos aspectos respecto a la forma de adquirir vocabulario. Al trabajar con la media, la función que más se ajusta es la lineal, con una pequeña meseta en el medio. Pensarla como forma de adquisición implica que, año tras año, los estudiantes están adquiriendo vocabulario momento tras momento, en el contacto con los textos académicos. Trabajar con la media y su evolución, que concuerda con la mediana en forma casi total, explica cerca de la totalidad de los datos, pero no puede dar cuenta del estado de amesetamiento de los 2 años intermedios ni del pronunciado ascenso del final.

Una aclaración a este dato lo puede dar la moda, que tiene la forma de una exponencial y explica que la adquisición de vocabulario tiene una etapa de crecimiento muy marcada hacia el final. La teoría del reacomodamiento conceptual permitiría explicar esta etapa de la adquisición. Pese a esto, este dato solo daría cuenta del crecimiento pronunciado del final, pero no del estancamiento del medio.

Para echar luz a este asunto, examinaremos, a continuación, los datos que se desprenden del análisis de los coeficientes de variaciones en las distintas transiciones anuales. En este aspecto, vamos a distinguir el valor de coeficiente de variación acumulado (que refleja el aumento de año tras año) del coeficiente de variación total (que refleja el aumento siempre respecto al primer año). 
Tabla 2.

Coeficiente de variación acumulado distribuido por años

\begin{tabular}{|c|c|c|c|}
\hline \multicolumn{4}{|c|}{ Coeficiente de variación acumulado } \\
\hline & \% C1-2 & \% C2-3 & \% C3-4 \\
\hline $\mathrm{x}$ & $23,53 \%$ & $3,37 \%$ & $17,95 \%$ \\
\hline $\mathrm{M}_{\mathrm{e}}$ & 10,71 & 1,70 & 13,79 \\
\hline $\mathrm{s}^{2}$ & 0,11 & 0,01 & 0,03 \\
\hline $\mathrm{s}$ & 0,33 & 0,08 & 0,18 \\
\hline
\end{tabular}

Fuente: Datos del autor.

Figura 4

Coeficiente de variación expresado como línea y función

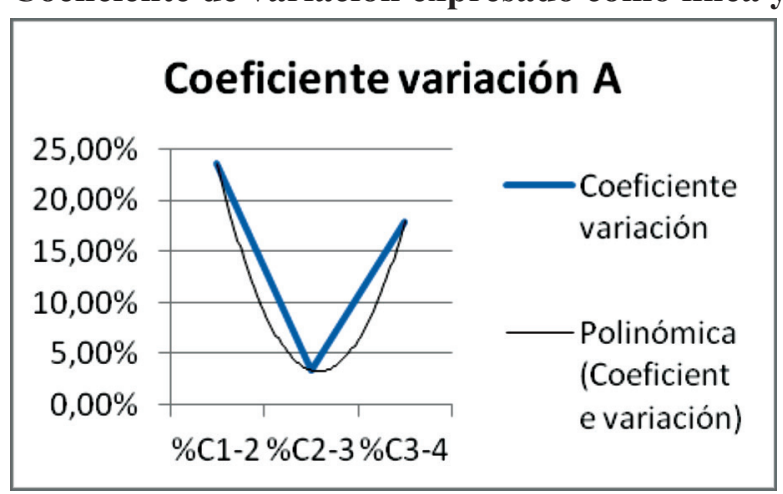

Fuente: Datos del autor.

Lo primero que nos permite interpretar el coeficiente de variación acumulado es que, dado que todos sus valores son positivos, en las 3 transiciones puede hablarse de aumento de vocabulario. Aún así, es llamativo el prácticamente ínfimo porcentaje de variación que encontramos en la transición A2-A3, y, si se suma el valor de la mediana en 1,70, encontramos una cantidad importante de valores negativos o muy cercanos a 0 , mostrando nada de adquisición o incluso una leve caída. Esto se vuelve especialmente cierto, al encontrar que las medidas de dispersión para estos valores son llamativamente bajas y reafirman la escasa mejora observada en este período. ¿Ahora por qué sucede esto, que número tras número se confirma, de un aprendizaje de vocabulario que se detiene por aproximadamente un año? Parecería que la adquisición de 
vocabulario puede darse por acumulación de información hasta cierto punto y, a partir de este, hace falta un reordenamiento conceptual, el ya mencionado insight, para dar otro salto cualitativo.

Los datos del coeficiente de variación total apoyan parcialmente esta idea.

Tabla 3.

Coeficiente de variación total

\begin{tabular}{|c|c|c|c|}
\hline \multicolumn{4}{|c|}{ Coeficiente de variación total } \\
\hline & \% C1-2 & \% C1-3 & \% C1-4 \\
\hline $\mathrm{x}$ & $23,53 \%$ & $26,88 \%$ & $50,49 \%$ \\
\hline $\mathrm{Me}$ & 0,11 & 0,15 & 0,42 \\
\hline $\mathrm{s} 2$ & 0,11 & 0,10 & 0,23 \\
\hline $\mathrm{s}$ & 0,33 & 0,32 & 0,48 \\
\hline
\end{tabular}

Fuente: Datos del autor.

Figura 5.

Coeficiente de variación total expresado como línea y función

\section{Coeficiente variación T}
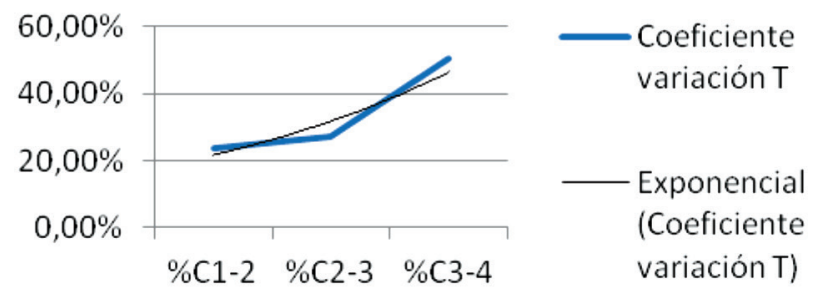

Fuente: Datos del autor.

Al contrastar los coeficientes siempre contra la medida inicial, podemos observar de una forma más absoluta cuánto es el aprendizaje en cada una de las instancias, no matizada por el valor anterior. Con una primera instancia no muy diferente a la segunda, y similares medidas de dispersión, ahora el amesetamiento o estancamiento podría ser analizado como una consecuencia neta del reordenamiento conceptual. 
Es decir, la idea de que en el primer intervalo hubo un gran incremento en la adquisición del vocabulario en realidad es un efecto de contraste con las pésimas condiciones iniciales, en términos de léxico, con las que empiezan los estudiantes. Es probable que parte del aumento en el primer intervalo se deba a la exposición y al trabajo con textos académicos, pero el motivo real del incremento se debe al reordenamiento conceptual inicial que sucede en los alumnos, quienes parten de una puntuación inicial muy baja y suben a una media similar al resto de la muestra en la segunda evaluación. A su vez, aquellos sujetos que partieron de una puntuación media o alta prácticamente no registran un aumento, sino hasta la última evaluación. Esta visión es plenamente consistente con una descripción exponencial del aprendizaje de vocabulario. Para verificar esta idea, presentamos, a continuación, los coeficientes de variación para 2 grupos: los que empezaron con una puntuación $<15$ y los que empezaron con una $>30$ (excluyendo los 3 casos que empezaron con puntuaciones de 40 , que no podrían registrar variación alguna).

Tabla 4.

Coeficiente de variación acumulado por puntuaciones en test

\begin{tabular}{|c|c|c|c|}
\hline \multicolumn{3}{|c|}{ Coeficiente de variación acumulado } \\
\hline$<15$ & $83,50 \%$ & $4,93 \%$ & $22,51 \%$ \\
\hline$>30$ & $1,75 \%$ & $2,91 \%$ & $5,79 \%$ \\
\hline$>25$ & $12,31 \%$ & $5,48 \%$ & $13,82 \%$ \\
\hline$>20$ & $24,59 \%$ & $6,03 \%$ & $21,48 \%$ \\
\hline
\end{tabular}

Fuente: Datos del autor.

Indudablemente, parte del poco coeficiente de incremento se debe a que una puntuación alta, como punto de partida, da un menor margen de crecimiento. Ahora, en ningún caso esta variable explica el enorme crecimiento que sucede en los grupos de baja puntuación inicial. ¿Por qué por mera exposición un grupo mejora su puntuación y otro no? Si la exposición fuera la única causa por la cual se obtiene vocabulario, en todos los grupos debería haber un incremento al menos similar al del grupo de menor rendimiento. Esto indudablemente pone en evidencia que hay un factor más allá del trabajo constante con material académico, y probablemente sea de orden conceptual, de reacomodamiento de 
ideas. Es decir, en los 2 grandes saltos de adquisición, estaría involucrado el reordenamiento, pero, para que suceda, se requiere una cantidad mínima de información. Esto explica también por qué el crecimiento que parece nulo para las primeras instancias de las puntuaciones altas se dispara en la última instancia.

\section{Conclusión}

Hemos realizado una descripción estadística y simple del aprendizaje de vocabulario a partir del tránsito que supone una carrera universitaria. A partir de este análisis, hemos encontrado ciertas regularidades en su adquisición. Para explicar esta última, que en primera instancia se ha descubierto como un hecho (lo cual refuerza aún más el papel de la educación en la conformación de vocabulario y no solo en un nivel superior o universitario, sino también secundario y fundamentalmente primario), hemos definido 2 hipótesis fundamentales que se podrían tener en cuenta.

La primera, y que es la sostenida por la mayoría de autores, es que el mero trabajo con diversos textos permite la incorporación de vocabulario. Esto parece ser parcialmente verdadero y, en este punto, hay que tener en cuenta que la mayoría de autores que sostiene esta hipótesis la aplican para el aprendizaje de una segunda o tercera lengua.

Si bien el trabajo con el texto es siempre necesario, pareciera ser que, en el caso del nivel superior, el texto es más bien una excusa, una base para que, a partir de ella, se produzca un reacomodamiento conceptual o ampliación de la memoria semántica del estudiante. Esto parece ser verdadero, en tanto la mejora en el aspecto productivo del vocabulario (lo que mide el test) requiere una base de lectura previa o puntuación base.

Finalmente, es preciso destacar que las puntuaciones observadas en la primera toma ponen de manifiesto un estado preocupante respecto a la competencia léxica, tanto general como productiva, de los jóvenes de primer año. A partir de esto, se debe promover la incorporación (que en este país ha empezado a ser ya tendencia) de espacios de trabajo de lecto-comprensión, en instancias previas al inicio de la carrera universitaria. 


\section{Referencias}

Álvarez Castrillo, C. y Diez Itza, E. (2000). Competencia léxica y rendimiento académico en alumnos de segundo año de bachillerato. Revista Aula Abierta, 76(1), 185-198.

Casso, J. (2010). Análisis y revisión críticas de los materiales de evaluación de la competencia léxica. Elaboración de un test de vocabulario de nivel umbral. Nebrija: Editorial del Departamento de Lenguas Aplicadas.

Carlino, P. (2007). ¿Qué nos dicen las investigaciones internacionales sobre escritura en la universidad? Cuadernos de Psicopedagogía, 4(1), 21-40.

Carlino, P. (2001). Hacerse cargo de la lectura y la escritura en la enseñanza universitaria de las ciencias sociales y humanas. Buenos Aires: Departamento de Educación, Universidad Nacional de Luján, Luján, Prov. de Buenos Aires.

Falco, M. y Kuz, A. (2016). Comprendiendo el Aprendizaje a través de las Neurociencias, con el entrelazado de las TICs en Educación. Buenos Aires: SEDICI.

Galagovsky Kurman, L. (2004). Del aprendizaje significativo al aprendizaje sustentable. Enseñanza de las Ciencias, 22(2), 229-240.

Galagovsky Kurman, L. (1996). Redes conceptuales: aprendizaje, comunicación y memoria. Buenos Aires: SYDALC.

Galagovsky Kurman, L. (1993). Redes conceptuales: base teórica e implicaciones para el proceso de enseñanza-aprendizaje. Enseñanza de las ciencias: Revista de Investigación y Experiencias Didácticas, 11(3), 301-307.

González, M. y Martínez, C. (1998). La enseñanza del vocabulario y el uso del diccionario. Encuentro. Revista de Investigación e Innovación en la clase de idiomas, 10(1), 1-10.

Manso, A. y Ballesteros, S. (2003). El papel de la agenda visoespacial en la adquisición del vocabulario ortográfico. Psicothema, 15(3), 388-394.

Martínez Fuentes, M. (1996). El papel del temperamento en la adquisición del lenguaje. Anales de Psicología, 12(2), 185-196.

Moya Guijarro, A. (2003). La adquisición/aprendizaje de la pronunciación, del vocabulario y de las estructuras interrogativas en lengua inglesa. Un estudio por edades. Didáctica, 15, 161-177. 
Nieto, D. (2005). Efectos del resumen. Sobre la comprensión lectora, la metacomprensión y el rendimiento académico. Revista de Educación, 337(1), 281-294.

Ocares, B., Reyes, F., Novoa, A., Véliz, M. y Castro, G. (2013). Competencia léxica, comprensión lectora y rendimiento académico en estudiantes de enseñanza media. Literatura y Lingüistica, 30(1), $165-180$.

Oster, U. (2009). La adquisición de vocabulario en una lengua extranjera: de la teoría a la aplicación didáctica. Revista Porta-Lingüarum, 11(1), 33-50.

Palma, B. y Cosmelli, D. (2008). Aportes de la Psicología y las Neurociencias al concepto del "Insight": La necesidad de un marco integrativo de estudio y desarrollo. Revista Chilena de Neuropsicología, 3(2), 14-27.

Rodriguez, M., Posada, M., Estrada, P. y Velasquez, M.. (2016). Población con riesgo de abandono universitario. Una aproximación desde la prevención. Antioquía: CLABES.

Serrano, C., Allegri, R. F., Drake, M., Butman, J., Harris, P. Nagle, C. y Ranalli, C.. (2001). Versión abreviada en español del test de denominación de Boston: su utilidad en el diagnóstico diferencial de la enfermedad de Alzheimer. Revista de Neurología, 33(7), 624-627.

Siguán, M. (2001). Bilingüismo y lenguas en contacto. La paz: Alianza editorial.

Suárez, Á., Moreno, J. y Godoy, M. (2010). Vocabulario y comprensión lectora: Algo más que causa y efecto. Álabe: Revista de Investigación sobre Lectura y Escritura, 1(1), 0-10.

Valdehíta, A. (2013). El efecto de tres actividades centradas en las formas: selección de definiciones, selección de ejemplos y la escritura de oraciones en el aprendizaje de segundas lenguas. RaeL: Revista Electrónica de Lingüística Aplicada, 12, 17-37.

Valdehíta, A. (2012). Aprendizaje de léxico en español como segunda lengua/lengua extranjera: Investigación sobre la efectividad de tres tipos de actividades para aprender vocabulario. (Disertación doctoral no publicada). España: UNED.

Vivas, J. et al. (2013). Psicología y otras ciencias del comportamiento. Compendio de investigaciones actuales I. Libertador San Martin: Editorial Universidad Adventista del Plata. 
Zapico, M. G. (2016). Evaluación de estrategias de enseñanza orientadas a competencias: una comparación de caso entre estilo orientado a la competencia y el orientado al contenido. Revista Ensayos Pedagógicos, XI(1), 135-146.

Zapico, M. G. (2016b). Evaluación de desempeño académico: la competencia léxica como competencia con validez predictiva. Revista Ensayos Pedagógicos, XI(2), 159-171.

Zapico, M. G. (2017). La competencia léxico-productiva como predictor del desempeño académico. Revista Ensayos Pedagógicos, XII(1), 123-138.

Zapico, M. G. (2017b). Sobre la necesidad de la enseñanza directa del vocabulario en los niveles primario y secundario del sistema educativo. Revista Ensayos Pedagógicos, XII(2), 17-31. 